\title{
Structural parameters of 11 faint Galactic globular clusters derived with 2MASS
}

\author{
C. Bonatto and E. Bica
}

\begin{abstract}
Universidade Federal do Rio Grande do Sul, Departamento de Astronomia CP 15051, RS, Porto Alegre 91501-970, Brazil e-mail: [charles;bica]@if.ufrgs.br
\end{abstract}

Received 17 September 2007 / Accepted 7 November 2007

\begin{abstract}
Context. Structural parameters and the total $M_{\mathrm{V}}$ magnitude are important properties for the characterisation of individual globular clusters. With the growth in statistics, especially of the intrinsically faint objects, the collective properties of the Galactic globular cluster system will be better defined, leading to a deeper understanding of the Galaxy formation processes.

Aims. We determine the structural parameters of 11 faint Galactic globular clusters that, in most cases, had not been previously studied in this context: IC 1257, Lyngå 7, Terzan 4, Terzan 10, BH 176, ESO 452-SC11, ESO 280-SC08, 2MASS-GC01, 2MASS-GC02, GLIMPSE-C01, and AL 3. They are projected not far from the central region of the Galaxy. Field-star contamination is significant in the colour-magnitude diagrams. Half of the sample has an absorption $A_{\mathrm{V}} \gtrsim 7$, in some cases reaching $A_{\mathrm{V}} \gtrsim 15$.

Methods. Stellar radial number-density and surface-brightness profiles were built with 2MASS photometry that, for the present clusters, corresponds to giant-branch stars. Field-star decontamination is essential for clusters in dense fields, so an algorithm that we had previously developed for open clusters in rich fields was employed to better define cluster sequences. With decontaminated photometry we also computed the total $M_{\mathrm{V}}$ of four such globular clusters, using M 4 as a template. King-like functions were fitted to the radial profiles, from which the core, half-light, half-star count, and tidal radii were derived, together with the concentration parameter. The derived parameters were then compared to equivalent ones available in the literature for other Galactic globular clusters.

Results. Compared to massive globular clusters, those in the present sample have smaller tidal and larger core radii for a given Galactocentric distance, which is consistent with rather loose structures. Globular cluster radii derived from number-density and surface-brightness profiles have similar values. The present magnitude estimates are $M_{\mathrm{V}} \approx-4.9(\mathrm{ESO} 280-\mathrm{SC} 08), M_{\mathrm{V}} \approx-5.8$ (2MASS-GC01), and $M_{\mathrm{V}} \approx-5.6$ (2MASS-GC02). We also estimate $M_{\mathrm{V}} \approx-6.1$ for GLIMPSE-C01, which is somewhat less luminous than previously given. The density profiles of Tz 4 and 2MASS-GC01 show evidence of post-core collapse clusters.

Conclusions. The structural parameters and luminosity of most of the faint globular clusters dealt with in this paper are consistent with those of Palomar-like (low mass and loose structure) globular clusters. This work helps to improve the coverage of the globular cluster parameter space.
\end{abstract}

Key words. Galaxy: globular clusters: general

\section{Introduction}

Astrophysical parameters of globular clusters (GCs) are of strong interest because they can be used to characterise GCs individually, eventually leading to a deeper understanding of their properties as a class in the Galaxy. From a broad perspective, such parameters may provide important clues to the investigation of the formation and evolution processes of the GC system of the Galaxy and elsewhere, e.g. Harris (1996, and the 2003 update $^{1}-$ hereafter H03), Mackey \& van den Bergh (2005), and Bica et al. (2006b). From the observational perspective, the depth of this kind of analysis directly depends on the statistical completeness of the sample, especially in the faint-GC tail, and on the reliability of the derived parameters.

In general terms, the standard picture of the GC structure assumes an isothermal central region and a tidally truncated outer region, usually described by a roughly spherical symmetry (e.g. Binney \& Merrifield 1998). In this context, the structure of most GCs is approximated well by a rather dense core and a sparse halo, but with a broad range in concentration. The most commonly used structural parameters are the core $\left(R_{\mathrm{c}}\right)$, half-light $\left(R_{\mathrm{hL}}\right)$, and tidal radii $\left(R_{\mathrm{t}}\right)$, along with the concentration

${ }^{1}$ http://physun.physics.mcmaster.ca/Globular.html parameter $c=\log \left(R_{\mathrm{t}} / R_{\mathrm{c}}\right)$, related to the isothermal sphere, single-mass model introduced by King (1962) to fit the surface brightness profiles (SBPs) of Galactic GCs.

As they age, GCs are continually harassed by external processes, such as tidal stress and dynamical friction (from the Galactic bulge and disk and from giant molecular clouds), and by internal ones, such as mass loss by stellar evolution, mass segregation, and low-mass star evaporation (e.g. Khalisi et al. 2007; Lamers et al. 2005; Gnedin \& Ostriker 1997). Over a Hubble time, these effects tend to lower the cluster mass, which may accelerate the core-collapse phase for some clusters (Djorgovski \& Meylan 1994, and references therein). As a dramatic end result, low-mass clusters may be completely disintegrated over the course of a few disruption time scales $\left(t_{\text {dis }}\right)$. Observational evidence for the dependence of $t_{\text {dis }}$ on cluster mass are given by, e.g. Janes \& Adler (1982), Wielen (1991), and Lamers et al. (2005). The last group of authors find $t_{\mathrm{dis}} \sim M^{0.62}$, which agrees with the $N$-body estimate of Baumgardt \& Makino (2003) for the disruption time scale due to the Galactic tidal field. Theoretically, the dependence of $t_{\text {dis }}$ on cluster mass was investigated by, e.g., Spitzer (1958) and Gnedin \& Ostriker (1997). Most of the above processes are related to the large-scale mass distribution of the Galaxy. Thus, their strength should depend on Galactocentric 
distance, and they are expected to be more effective for the lessmassive clusters (e.g. Djorgovski \& Meylan 1994; van den Bergh 1991; Chernoff \& Djorgovski 1989). Consequently, the distribution of structural parameters among the Galactic GC population, as well as their dependence on Galactocentric distance, may also be related to the physical conditions prevailing at the early formation phase (e.g. van den Bergh 1991).

Considering all these arguments, it is natural to assume that the long-term (more than $10 \mathrm{Gyr}$, in most cases) dynamical evolution of the collective GC population (as well as the individual GCs) may be reflected in the statistical properties of their structural parameters. It is in this context that intrinsically faint GCs play an important rôle, since they are more sensitive to the processes discussed above and have shorter dynamical-evolution time scales than the massive ones.

In most cases, the SBPs of faint GCs may contain significant noise owing to the intrinsically-low surface brightness outside the central region, and because of the random presence of relatively bright stars, either from the field or cluster members. The same applies to the outer parts, where background starlight is usually a major contributor, even in luminous GCs. Structural parameters measured in such SBPs would certainly be affected to varying degrees. In such cases, the alternative is to work with stellar radial density profiles (RDPs), in which only the projected number-density of stars is taken into account, irrespective of the individual stellar brightness. RDPs are particularly appropriate for the outer parts, provided that a statistically significant, and reasonably uniform, comparison field is available to tackle the background contamination. Such wide fields are provided by 2MASS (e.g. Bonatto \& Bica 2005; Bonatto \& Bica 2007a). However, the 2MASS photometric depth limit implies that the giant branch can be accessed in GCs more distant than a few kpc from the Sun (Bonatto \& Bica 2007b). Thus, it is necessary to take depth-limited effects into account when considering such structural parameters in the context of absolute comparisons using parameters obtained with deeper photometry. This issue was studied in Bonatto \& Bica (2007b) by means of model GCs built assuming different conditions, which allowed us to investigate relations among radii measured in RDPs and SBPs, together with their dependence on photometric depth.

Trager et al. (1995) presented a catalogue of SBPs for 125 Galactic GCs with structural parameters derived mostly with the King (1966) profile. Based mostly on the earlier work, H03 provide structural parameters for 141 (of the total catalogued 150 at the time) GCs. Recently, Cohen et al. (2007) have used surface brightness profiles in $J, H$, and $K_{\mathrm{s}}$ with $2 \mathrm{MASS}^{2}$ photometry to derive core radii for $104 \mathrm{GCs}$, by means of fits of a power-law with a core function. The 9 remaining GCs in H03 are faint and were not included in Cohen et al. (2007).

The compilation of $\mathrm{H} 03$ contains $150 \mathrm{GCs}$ but, as a consequence of recent deeper surveys, the number of known GCs in the Galaxy has been increasing. For instance, Kobulnicky et al. (2005) discovered the far-IR GC GLIMPSE-C01. Carraro (2005) identified Whiting 1 as a young halo GC. Two stellar systems detected with the Sloan Digital Sky Survey (SDSS) in the outer halo, Willman 1 (SDSS J1049+5103) and SDSS J1257+3419, might be GCs or dwarf galaxies (Willman et al. 2005; Sakamoto \& Hasegawa 2006). Ortolani et al. (2006) identified AL 3 as a new GC in the bulge with a blue horizontal branch. Next, Froebrich et al. (2007) found evidence that FSR 1735 is an inner Galaxy GC, and Belokurov et al. (2007) found the faint halo GC SEGUE1 using SDSS. More recently,

2 http://www.ipac.caltech.edu/2mass/releases/allsky/
Koposov et al. (2007) have reported the discovery of two verylow luminosity halo GCs (Koposov 1 and 2) detected with SDSS. The last three GCs are beyond the scope of the present paper because they are too distant and/or exceedingly faint. Another object, FSR 0584, has recently been identified as a nearby Palomarlike halo GC or an old open cluster (OC) by Bica et al. (2007). Finally, Bonatto et al. (2007) have identified FSR 1767 as a bulge-projected $\left(\ell=352.6^{\circ}, b=-2.17^{\circ}\right)$ Palomar-like GC $\left(M_{\mathrm{V}} \approx-4.7\right)$ that appears to be the nearest one $\left(d_{\odot} \approx 1.5 \mathrm{kpc}\right)$ so far discovered. Fundamental parameters and updates to the GCs in H03 were provided by Bica et al. (2006b), also available as a Vizier on-line catalogue ${ }^{3}$.

The main goal of the present work is to derive structural parameters, using RDPs and SBPs built with 2MASS star-counts and photometry, for the remaining 9 faint GCs in H03, which lack this kind of data. The targets are IC 1257, Lyngå 7, Terzan 4, Terzan 10, BH 176, ESO 452-SC11, ESO 280-SC08, 2MASSGC01, and 2MASS-GC02. We also include in the analyses the more recently discovered GCs GLIMPSE-C01 (Kobulnicky et al. 2005) and AL 3 (Ortolani et al. 2006).

This paper is structured as follows. In Sect. 2 we present previous results on the present GCs that are relevant for this work. In Sect. 3 we describe the 2MASS photometry, including extraction and errors, build the near-infrared colour-magnitude diagrams (CMDs) for the present GCs, briefly discuss the tools and algorithms employed to decontaminate the CMDs of field stars, and estimate the absolute $V$ magnitudes of ESO 280-SC06, 2MASS-GC01, 2MASS-GC02, and GLIMPSE-C01. In Sect. 4 we build RDPs and SBPs and derive the core, half-light, half-star count, and tidal radii, together with the concentration parameter for the present GC sample. In Sect. 5 we discuss the potential effects of depth-limited photometry in the derivation of structural parameters from radial profiles and compare such parameters with those of other globular clusters in the Galaxy. Finally, concluding remarks are given in Sect. 6.

\section{The faint GC sample}

In the following we recall fundamental literature results for the present analyses.

IC 1257: This object was first classified as an OC (Lyngå 1987, and references therein), but Harris et al. (1997) identified it as a GC using $B, V$, and $I$ photometry. They also determined the cluster reddening $E(B-V)=0.75$, distance from the Sun $d_{\odot}=$ $24 \mathrm{kpc}$, and metallicity $[\mathrm{Fe} / \mathrm{H}]=-1.7$. Its absolute magnitude is $M_{\mathrm{V}}=-6.15(\mathrm{H} 03)$.

Lyngå 7: Lyngå (1964) discovered and classified Lyngå 7 as a Trumpler type II 2 p OC, which was later reported in Alter et al. (1970). Ortolani et al. (1993) identified it as a GC with $B, V$, and $I$ photometry and determined $E(B-V)=0.72,[\mathrm{Fe} / \mathrm{H}]=$ -0.40 and $d_{\odot}=6.7 \mathrm{kpc}$. Tavarez \& Friel (1995) used moderate resolution spectroscopy to obtain a radial velocity $V_{\mathrm{R}}=$ $6 \pm 15 \mathrm{~km} \mathrm{~s}^{-1},[\mathrm{Fe} / \mathrm{H}]=-0.62, E(B-V)=0.70$, and $d_{\odot}=$ $7.2 \mathrm{kpc}$. Based on 2MASS photometry, Sarajedini (2004) derived $[\mathrm{Fe} / \mathrm{H}]=-0.76$ and $d_{\odot}=7.3 \mathrm{kpc}$ by adopting $E(B-V)=$ 0.73 .

\footnotetext{
3 http://vizier.cfa.harvard.edu/viz-bin/VizieR? - source $=$ J $/ A+A / 450 / 105$
} 
Terzan 4: This GC was discovered by Terzan (1971). Based on $V, I$, and Gunn $\mathrm{Z}$ photometry, Ortolani et al. (1997) derived $E(B-V)=2.35, d_{\odot}=8.3 \mathrm{kpc}$ and $[\mathrm{Fe} / \mathrm{H}]=-2.0$. Origlia \& Rich (2004) obtained $[\mathrm{Fe} / \mathrm{H}]=-1.6$ using high-dispersion spectroscopy. Ortolani et al. (2007) derived $d_{\odot}=8.0 \mathrm{kpc}$ from HSTNICMOS photometry. Its absolute magnitude is $M_{\mathrm{V}}=-6.09$ ( $\mathrm{H} 03)$.

Terzan 10: This is another GC discovered by Terzan (1971). Ortolani et al. (1997) derived $E(B-V)=2.40, d_{\odot}=4.8 \mathrm{kpc}$, and $[\mathrm{Fe} / \mathrm{H}]=-1.0$ from $V, I$, and Gunn $\mathrm{Z}$ photometry. Its absolute magnitude is $M_{\mathrm{V}}=-6.31(\mathrm{H} 03)$.

BH 176: It was discovered by van den Bergh \& Hagen (1975). Ortolani et al. (1995) obtained $E(B-V)=0.77, d_{\odot}=13.4 \mathrm{kpc}$, and a nearly solar metallicity by means of $V$ and $I$ photometry. They emphasise that, owing to its low Galactic latitude and high metallicity, the nature of $\mathrm{BH} 176$ as a metal-rich GC or an old OC was not clear. Phelps \& Schick (2003) obtained an age of $7 \mathrm{Gyr}, E(B-V)=0.53, d_{\odot}=18 \mathrm{kpc}$, and $[\mathrm{Fe} / \mathrm{H}]=0.0$ with $V$ and $I$ photometry. They point out that that the cluster might be an old metal-rich OC or a young metal-rich GC. Another possibility is that BH 176 is associated with an accreted galaxy event now related to the Galactic anticentre stellar structure or the Monoceros Ring (Frinchaboy 2006). H03 provides the absolute magnitude $M_{\mathrm{V}}=-4.35$.

ES0 452-SC11: It was discovered and classified as a GC by Lauberts (1981). Bica et al. (1999) confirmed it as a GC and determined $E(B-V)=0.58, d_{\odot}=6.5 \mathrm{kpc}$, and $[\mathrm{Fe} / \mathrm{H}]=-1.5$ from $V$ and $I$ photometry. Using $V$ and $I$ photometry Cornish et al. (2006) found $E(B-V)=0.57, d_{\odot}=7.1 \mathrm{kpc}$, and $[\mathrm{Fe} / \mathrm{H}]=$ -1.15 . ESO 452-SC11 is intrinsically faint, with $M_{\mathrm{V}}=-3.97$ ( $\mathrm{H} 03)$.

ESO 280-SC06: Lauberts (1982) reported and classified ESO 280-SC06 as an OC. Ortolani et al. (2000) identify it as a $\mathrm{GC}$, and also obtained $E(B-V)=0.07,[\mathrm{Fe} / \mathrm{H}]=-1.8$, and $d_{\odot}=21.9 \mathrm{kpc}$ from $V$ and $I$ photometry.

2MASS-GCQ1: The infrared 2MASS-GC01 was discovered by Hurt et al. (2000). Ivanov et al. (2000) obtained $E(B-V)=$ 6.74 and $d_{\odot}=3.1 \mathrm{kpc}$ from NTT near IR photometry. Ivanov $\&$ Borissova (2002) obtained $[\mathrm{Fe} / \mathrm{H}]=-1.19$ from $2 \mathrm{MASS}$ photometry.

2MASS-GCQ2: The infrared GC 2MASS-GC02 was discovered by Hurt et al. (2000). Ivanov et al. (2000) obtained $E(B-V)=$ $5.55, d_{\odot}=3.9 \mathrm{kpc}$, and $[\mathrm{Fe} / \mathrm{H}]=-0.66$ from NTT near IR-photometry.

GLIMPSE-CQ1: The infrared GC GLIMPSE-C01 was discovered by Kobulnicky et al. (2005), who obtained a kinematic distance of $3.1-5.2 \mathrm{kpc}, R_{\mathrm{c}}=30^{\prime \prime}$, and $R_{\mathrm{hl}}=36^{\prime \prime}$ and estimated a total magnitude $M_{\mathrm{V}}=-8.4 \pm 3$. Ivanov et al. (2005) derived $E(B-V)=5.0, d_{\odot}=3.7 \mathrm{kpc}$ and $[\mathrm{Fe} / \mathrm{H}]=-1.61$ from NTT near IR photometry. They also obtained the structural parameters $R_{\mathrm{c}}=0.84 \mathrm{pc}\left(\approx 0.76^{\prime}\right)$ and $R_{\mathrm{t}}=29 \mathrm{pc}\left(\approx 26^{\prime}\right)$, which we compare with the present values (Sect. 4 ). These cluster radii (especially the tidal) were estimated by extrapolation, since they worked with NTT/Sofi photometry that covers an area of $4.92^{\prime} \times 4.92^{\prime}$ $\left(\approx 5 \times 5 \mathrm{pc}^{2}\right)$, which does not reach the tidal radius.

AL 3: This cluster was discovered by Andrews \& Lindsay (1967) and also catalogued as BH 261 by van den Bergh \& Hagen (1975). In these studies it was classified as an OC. Ortolani et al. (2006) identify it as a GC with $B, V$, and $I$ photometry and derive $E(B-V)=0.36, d_{\odot}=6.0 \mathrm{kpc},[\mathrm{Fe} / \mathrm{H}]=-1.3$, a half-density radius $R_{\mathrm{hd}}=2.2 \mathrm{pc}$, and $M_{\mathrm{V}}=-4.0$.

Table 1 summarises the fundamental data for the sample GCs. It also contains absolute magnitude estimates derived in this work (Sect. 3.4) for some GCs that lacked this information. For GLIMPSE-C01 we include an additional row that gives our estimate of $M_{\mathrm{V}}$.

\section{2MASS photometry and CMD analytical tools}

In recent years we have developed a series of tools to statistically disentangle field and cluster stars that have been applied to objects in a wide variety of environmental conditions. They are based on wide-field 2MASS photometry and essentially deal with CMDs and stellar RDPs. As a result, CMDs containing more enhanced cluster sequences, and RDPs with a significant contrast with the background were obtained, which in turn, allowed us to derive more constrained cluster fundamental and structural parameters. For instance, nearby OCs were analysed in detail like NGC 3960 and M52 (Bonatto \& Bica 2006), and NGC 4755 (Bonatto et al. 2006b), embedded clusters like NGC 6611 (Bonatto et al. 2006a), and relatively distant OCs like BH63 (Bica et al. 2006a). These tools are explained in Bonatto \& Bica (2007a), where we studied relatively distant OCs in heavily contaminated fields towards the bulge and/or low-disk directions.

With respect to cluster structure, most of our analysis is based on large-scale radial profiles, which are fundamental to derive structural parameters of star clusters, the tidal radius in particular. Such profiles can be obtained using the uniform, allsky photometric coverage provided by 2 MASS (e.g. Bonatto \& Bica 2007a, 2005).

\subsection{MASS extractions}

The present faint GCs were analysed with $J, H$, and $K_{\mathrm{s}} 2 \mathrm{MASS}$ photometry extracted in circular fields of radius $R_{\text {ext }}$ (Table 1) centred on the coordinates of the objects (Table 1) using VizieR ${ }^{4}$. Our experience with OC analysis (e.g. Bonatto \& Bica 2007a, and references therein) shows that, as long as no other populous cluster is present in the field and differential absorption is not prohibitive, wide extraction areas can provide the required stellar statistics, in terms of magnitude and colours, for a consistent field-star decontamination (Sect. 3.2). Working with wide comparison fields also results in stellar radial profiles with more enhanced contrast with the background (Sect. 4).

Based on our experience with the analysis of clusters in crowded field directions using 2MASS photometry (Bonatto \& Bica 2007a, and references therein), the upper limit for the $J, H$, and $K_{\mathrm{s}}$ photometric uncertainties in the 2MASS extractions was adopted as $0.5 \mathrm{mag}$. This condition represents a compromise between statistically significant star counts and photometric quality. Note that about $75 \%-85 \%$ of the extracted stars have errors

\footnotetext{
4 vizier.u-strasbg.fr/viz-bin/VizieR?-source=II/246
} 
Table 1. Fundamental parameters in the literature and 2MASS extraction radius.

\begin{tabular}{|c|c|c|c|c|c|c|c|c|c|c|}
\hline GC & $\begin{array}{c}\alpha(2000) \\
(\mathrm{hms})\end{array}$ & $\begin{array}{c}\delta(2000) \\
\left({ }^{\prime \prime \prime \prime}\right)\end{array}$ & $\begin{array}{r}\ell \\
\left({ }^{\circ}\right)\end{array}$ & $\begin{array}{r}b \\
\left(^{\circ}\right)\end{array}$ & $\begin{array}{r}R_{\text {ext }} \\
\left({ }^{\prime}\right)\end{array}$ & $M_{\mathrm{V}}$ & $E(B-V)$ & {$[\mathrm{Fe} / \mathrm{H}]$} & $\begin{array}{r}d_{\odot} \\
(\mathrm{kpc})\end{array}$ & $\begin{array}{r}R_{\mathrm{GC}} \\
(\mathrm{kpc})\end{array}$ \\
\hline (1) & (2) & (3) & (4) & (5) & (6) & (7) & (8) & (9) & (10) & (11) \\
\hline IC 1257 & $17: 27: 58$ & $-07: 05: 35$ & 16.53 & +15.15 & 60 & $-6.15^{* *}$ & 0.73 & -1.70 & 25.0 & 18.5 \\
\hline Lyngä 7 & 16:11:03 & $-55: 18: 52$ & 328.77 & -2.79 & 40 & $-6.37^{\dagger}$ & 0.73 & -0.62 & 7.2 & 3.9 \\
\hline $\mathrm{Tz} 4$ & $17: 30: 34$ & $-31: 35: 44$ & 356.02 & +1.31 & 40 & $-6.09^{* *}$ & 2.35 & -1.60 & 9.1 & 2.0 \\
\hline $\mathrm{Tz} 10$ & 18:02:57 & $-26: 04: 00$ & 4.42 & -1.86 & 40 & $-6.31^{* *}$ & 2.40 & -1.00 & 5.7 & 1.6 \\
\hline BH 176 & 15:39:07 & $-50: 03: 02$ & 328.41 & +4.34 & 40 & $-4.35^{* *}$ & 0.77 & 0.00 & 15.6 & 10.2 \\
\hline ESO 452-SC11 & $16: 39: 26$ & $-28: 23: 52$ & 351.91 & +12.10 & 60 & $-3.97^{* *}$ & 0.49 & -1.50 & 7.8 & 2.0 \\
\hline ESO 280-SC06 & 18:09:12 & $-46: 24: 00$ & 346.93 & -12.58 & 50 & $-4.9 \pm 0.3^{*}$ & 0.07 & -1.80 & 21.7 & 15.0 \\
\hline 2MASS-GC01 & 18:08:22 & $-19: 49: 47$ & 10.47 & +0.10 & 40 & $-5.8 \pm 0.2^{*}$ & 6.80 & -1.19 & 3.1 & 4.2 \\
\hline 2MASS-GC02 & 18:09:37 & $-20: 46: 44$ & 9.78 & -0.62 & 40 & $-5.6 \pm 0.2^{*}$ & 5.56 & -0.66 & 3.9 & 3.4 \\
\hline GLIMPSE-C01 & $18: 48: 51$ & $-01: 29: 50$ & 31.30 & -0.10 & 60 & $-8.4 \pm 3^{\ddagger}$ & 5.00 & -1.60 & 3.7 & 4.5 \\
\hline GLIMPSE-C01 & & & & & & $-6.1 \pm 0.2^{*}$ & & & & \\
\hline AL 3 & 18:14:06 & $-28: 38: 12$ & 3.36 & -5.27 & 40 & $-4.00^{* *}$ & 0.36 & -1.30 & 6.0 & 1.4 \\
\hline
\end{tabular}

Notes. Columns 2 and 3: central coordinates from H03. Column 6: 2MASS extraction radius. **: $M_{\mathrm{V}}$ from H03; *: $M_{\mathrm{V}}$ estimated by comparison with M 4 (Sect. 3.4); ${ }^{\dagger}: M_{\mathrm{V}}$ from Bica et al. (2006b). ${ }^{\ddagger}: M_{\mathrm{V}}$ from Kobulnicky et al. (2005). Column 10: distance from the Sun from Bica et al. (2006b). Column 11: Galactocentric distance computed using $R_{\mathrm{GC}}=7.2 \mathrm{kpc}$ (Bica et al. 2006b) as the distance of the Sun to the Galactic centre. All additional information is from $\mathrm{H} 03$.

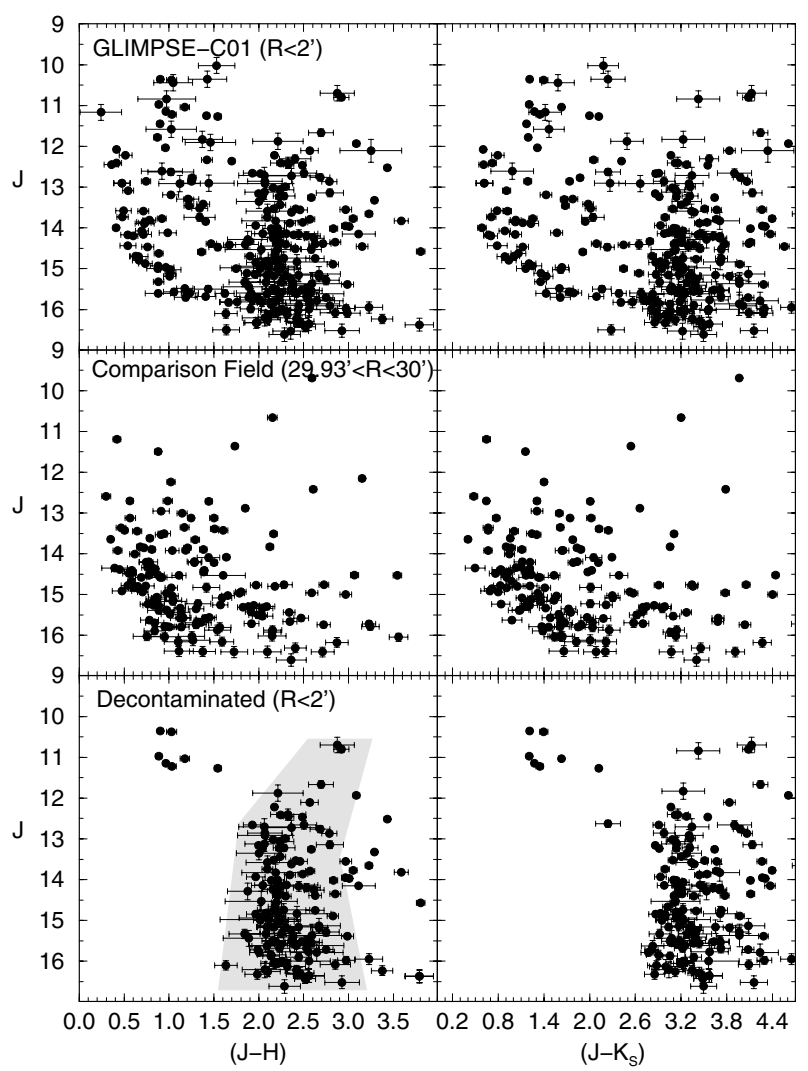

Fig. 1. 2MASS CMDs extracted from the $R<2^{\prime}$ region of GLIMPSE-C01. Top panels: observed photometry with the colours $J \times(J-H)($ left $)$ and $J \times\left(J-K_{\mathrm{s}}\right)($ right $)$. Middle: equal-area comparison field showing that the dominant contamination, in this case, arises from disk stars. Bottom panels: field star decontaminated CMDs. The colour-magnitude filter used to isolate probable cluster stars (Sect. 3.3) is shown as a shaded region.

smaller than $0.06 \mathrm{mag}$ in the three 2MASS bands. A typical distribution of 2MASS errors as a function of magnitude, for clusters at approximately the same central directions as the present

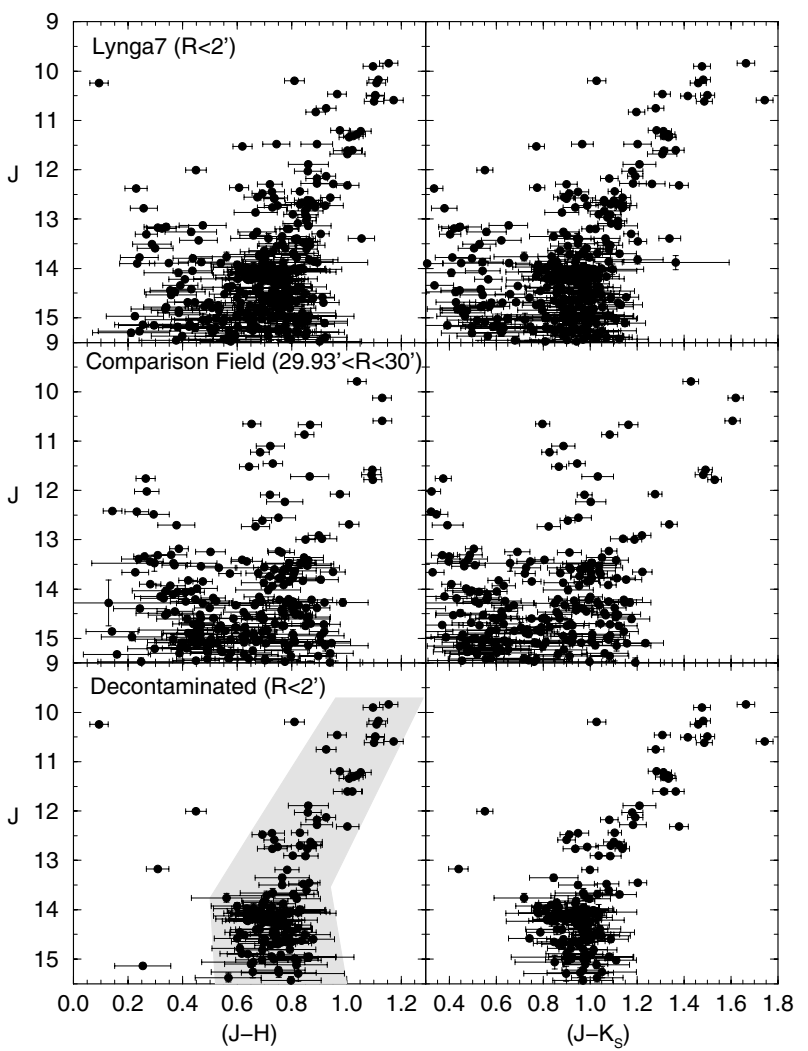

Fig. 2. Same as Fig. 1 for the $R<2^{\prime}$ region of Lyngå 7 .

GCs, can be found in Bonatto \& Bica (2007a). The relatively small size of error bars, propagated to the colours, can be appreciated in the actual CMDs of the present sample GCs shown in Figs. 1-3.

We illustrate as CMD analyses GLIMPSE-C01 (Fig. 1) and Lyngå 7 (Fig. 2), which are projected $\approx 30^{\circ}$ from the direction of the centre (Table 1). 2MASS $J \times(J-H)$ and $J \times\left(J-K_{\mathrm{s}}\right)$ CMDs extracted from a central $\left(R<2^{\prime}\right)$ region of both GCs (top panels) can be contrasted with the respective equal-area CMDs 


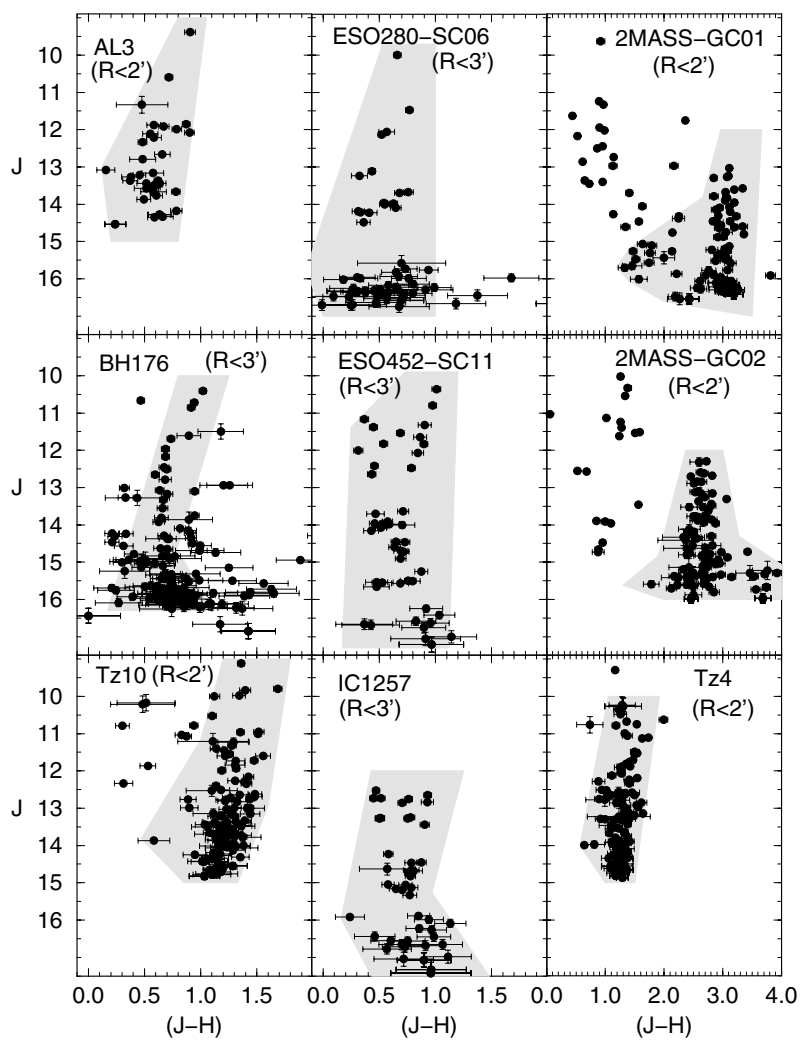

Fig. 3. Decontaminated $J \times(J-H)$ CMDs of the remaining GCs. The colour-magnitude filters are shown as a shaded region.

extracted from the comparison field (middle panels). It is clear that the main contaminant are disk stars in both cases, with some contribution from bulge stars.

\subsection{Field-star decontamination}

To disentangle the intrinsic CMD morphology from the field, we apply the statistical decontamination algorithm described in Bonatto \& Bica (2007a). It measures the relative numberdensities of candidate cluster and field stars in small cubic CMD cells with axes corresponding to the magnitude $J$ and the colours $(J-H)$ and $\left(J-K_{\mathrm{s}}\right)$ (considering also the $1 \sigma$ uncertainties in the 2MASS bands). These colours provide the maximum discrimination among CMD sequences for star clusters of different ages (e.g. Bonatto et al. 2004).

Basically, the algorithm (i) divides the full range of magnitude and colours of a CMD into a 3D grid, (ii) computes the expected number-density of field stars in each cell based on the number of comparison field stars with magnitude and colours compatible with those in the cell, and (iii) subtracts the expected number of field stars from each cell. Typical cell dimensions are $\Delta J=0.5$, and $\Delta(J-H)=\Delta\left(J-K_{\mathrm{s}}\right)=0.25$, which are large enough to allow sufficient star-count statistics in individual cells and small enough to preserve the morphology of the CMD evolutionary sequences. As comparison field we use the region within the respective tidal (Table 2) and extraction (Table 1) radii to obtain representative background statistics.

The algorithm is applied for 3 different grid specifications in each dimension to minimise potential artifacts introduced by the choice of parameters, thus resulting in 27 different outputs. The average number of probable cluster stars $\left\langle N_{\mathrm{cl}}\right\rangle$ is computed from these outputs. Typical standard deviations of $\left\langle N_{\mathrm{cl}}\right\rangle$ are at the $\approx 2.5 \%$ level. The final field star-decontaminated CMD contains the $\left\langle N_{\mathrm{cl}}\right\rangle$ stars with the highest number frequencies. Stars that remain after the decontamination are in cells where the stellar density presents a clear excess over the field. Consequently, they have a significant probability of being cluster members. More details on the algorithm, including discussions on subtraction efficiency and limitations, are given in Bonatto \& Bica (2007a).

The resulting decontaminated CMDs of GLIMPSE-C01 and Lyngå 7 are shown in the bottom panels of Figs. 1 and 2. As expected, most of the disk component is removed, leaving a sequence of stars that corresponds essentially to the giant branch.

For the sake of space, only the decontaminated $J \times(J-H)$ CMDs of the remaining GCs are shown in Fig. 3. In all cases, the spatial region shown in the CMDs lies within the respective half-star count radius (Table 2).

Differential absorption appears to be similar in each GC and in respective field extractions, as indicated, for instance, by GLIMPSE-C01 (Fig. 1), which is affected by a high reddening value. The similarity provides a consistent field subtraction, as suggested by the decontaminated resulting giant branches in Fig. 3. Actually, differential absorption, when present, is more critical for low-density star clusters (e.g. the open cluster NGC 6611, Bonatto et al. 2006a), especially in the optical.

\subsection{Colour-magnitude filters}

Decontaminated CMDs present better-defined cluster sequences. Based on these sequences, more intrinsic colour-magnitude filters can be designed to remove stars with colours compatible with those of the foreground/background field, which in turn improves the cluster/background contrast. They are wide enough to accommodate the colour distribution of cluster CMD sequences, allowing for $1 \sigma$ uncertainties. However, residual field stars with colours similar to those of the cluster are expected to remain within the colour-magnitude filter. This residual contamination is statistically evaluated by means of the comparison field. Colour-magnitude filter widths should also account for formation or dynamical evolution-related effects, such as enhanced fractions of binaries (and other multiple systems) towards the central parts of clusters, since such systems tend to widen the sequences (e.g. Bonatto \& Bica 2007a; Bonatto et al. 2005; Hurley $\&$ Tout 1998; Kerber et al. 2002).

The colour-magnitude filters for the present GCs are shown in Figs. 1-3 as a shaded region superimposed on the field-star decontaminated CMDs. In the present cases, they basically isolate giant-branch stars. As a rule, the filters are wide enough to take all the relevant stars into account in both colours. Thus, we only apply the method to the $(J-H)$ colour.

\subsection{Absolute magnitude estimates}

ESO 280-SC06, 2MASS-GC01, and 2MASS-GC02 lack absolute magnitudes, while the available estimate for GLIMPSE-C01 has a large uncertainty (Table 1). Here, such values are estimated following a similar method to the one used for the recently identified GC FSR 1767 (Bonatto et al. 2007). Basically, we compare the number of giant stars in ESO 280-SC06, 2MASS-GC01, 2MASS-GC02, and GLIMPSE-C01 with that in the nearby $\left(d_{\odot} \approx 2.2 \mathrm{kpc}\right)$, intermediate-metallicity $([\mathrm{Fe} / \mathrm{H}]=-1.2)$ and total absolute magnitude $M_{\mathrm{V}}=-7.2 \mathrm{GC} \mathrm{M} 4$ (H03, and references therein). In all cases, including M4, the number of member giant stars is computed from decontaminated 2MASS 
Table 2. Structural parameters derived from 2MASS data.

\begin{tabular}{|c|c|c|c|c|c|c|c|c|c|}
\hline \multirow[b]{2}{*}{$\mathrm{GC}$} & \multirow[b]{2}{*}{$\begin{array}{c}1^{\prime} \\
(\mathrm{pc})\end{array}$} & \multicolumn{4}{|c|}{ RDP } & \multicolumn{4}{|c|}{ SBP } \\
\hline & & $\begin{array}{l}R_{\mathrm{c}} \\
\left({ }^{\prime}\right)\end{array}$ & $\begin{array}{c}R_{\mathrm{hSC}} \\
\left(^{\prime}\right)\end{array}$ & $\begin{array}{l}R_{\mathrm{t}} \\
\left({ }^{\prime}\right)\end{array}$ & $\mathrm{c}$ & $\begin{array}{l}R_{\mathrm{c}} \\
\left({ }^{\prime}\right)\end{array}$ & $\begin{array}{c}R_{\mathrm{hL}} \\
\left({ }^{\prime}\right)\end{array}$ & $\begin{array}{l}R_{\mathrm{t}} \\
\left({ }^{\prime}\right)\end{array}$ & $\mathrm{c}$ \\
\hline (1) & (2) & (3) & (4) & (5) & (6) & (7) & (8) & (9) & (10) \\
\hline IC 1257 & 7.3 & $0.2 \pm 0.1$ & $1.4 \pm 0.3$ & $7.1 \pm 2.0$ & $1.5 \pm 0.2$ & $0.3 \pm 0.1$ & $1.4 \pm 0.2$ & $10.7 \pm 2.8$ & $1.6 \pm 0.2$ \\
\hline Lyngä 7 & 2.1 & $0.9 \pm 0.2$ & $1.2 \pm 0.1$ & $8.4 \pm 1.2$ & $1.0 \pm 0.1$ & $0.9 \pm 0.3$ & $1.2 \pm 0.3$ & $7.7 \pm 1.4$ & $0.9 \pm 0.2$ \\
\hline Tz4 & 2.6 & $0.8 \pm 0.4$ & $1.9 \pm 0.2$ & $6.2 \pm 1.4$ & $0.9 \pm 0.2$ & $1.0 \pm 0.4$ & $1.8 \pm 0.2$ & $7.6 \pm 1.3$ & $0.9 \pm 0.2$ \\
\hline Tz 10 & 1.7 & $1.0 \pm 0.3$ & $1.6 \pm 0.2$ & $5.9 \pm 2.0$ & $0.8 \pm 0.2$ & $0.8 \pm 0.4$ & $1.5 \pm 0.2$ & $3.4 \pm 0.8$ & $0.7 \pm 0.3$ \\
\hline BH 176 & 4.5 & $0.9 \pm 0.2$ & $1.0 \pm 0.1$ & $7.6 \pm 1.1$ & $0.9 \pm 0.1$ & $0.9 \pm 0.2$ & $0.8 \pm 0.1$ & $5.9 \pm 0.5$ & $0.8 \pm 0.1$ \\
\hline ESO 452-SC11 & 2.3 & $0.5 \pm 0.2$ & $0.5 \pm 0.2$ & $5.4 \pm 1.8$ & $1.0 \pm 0.2$ & $0.5 \pm 0.2$ & $0.5 \pm 0.2$ & $4.8 \pm 1.2$ & $1.0 \pm 0.2$ \\
\hline ESO 280-SC06 & 6.3 & $0.7 \pm 0.2$ & $1.1 \pm 0.2$ & $5.3 \pm 0.9$ & $0.9 \pm 0.1$ & $0.5 \pm 0.2$ & $1.0 \pm 0.2$ & $5.0 \pm 1.1$ & $0.9 \pm 0.2$ \\
\hline 2MASS-GC01 & 0.9 & $1.0 \pm 0.2$ & $1.7 \pm 0.2$ & $7.5 \pm 1.3$ & $0.9 \pm 0.1$ & $0.7 \pm 0.2$ & $1.6 \pm 0.2$ & $4.4 \pm 0.4$ & $0.8 \pm 0.2$ \\
\hline 2MASS-GC02 & 1.1 & $0.6 \pm 0.1$ & $0.6 \pm 0.2$ & $4.8 \pm 0.6$ & $0.9 \pm 0.1$ & $0.4 \pm 0.1$ & $0.5 \pm 0.2$ & $3.7 \pm 0.4$ & $1.0 \pm 0.1$ \\
\hline GLIMPSE-C01 & 1.1 & $0.4 \pm 0.1$ & $0.8 \pm 0.1$ & $8.0 \pm 1.0$ & $1.3 \pm 0.1$ & $0.4 \pm 0.1$ & $0.5 \pm 0.1$ & $4.5 \pm 0.5$ & $1.1 \pm 0.1$ \\
\hline AL 3 & 1.7 & $0.4 \pm 0.3$ & $0.5 \pm 0.2$ & $4.1 \pm 0.5$ & $1.0 \pm 0.3$ & $(\dagger)$ & $0.6 \pm 0.2$ & $(\dagger)$ & $(\dagger)$ \\
\hline
\end{tabular}

Notes. Column 2: arcmin to parsec scale. Columns 4 and 8: half-star count and half-light radii, respectively. Columns 6 and 10: concentration parameter, $c=\log \left(R_{\mathrm{t}} / R_{\mathrm{c}}\right) .(\dagger)$ : the SBP of AL 3 could not be fitted because of exceeding noise, especially beyond $R=1^{\prime}$.

photometry (Sect. 3.2), considering stars in the region from the cluster centre to the tidal radius.

We assume that the decontaminated giants in ESO 280SC06, 2MASS-GC01, 2MASS-GC02, and GLIMPSE-C01 are roughly of the same spectral type as in $\mathrm{M} 4$, and also that the luminosity of a typical GC is dominated by the number of giants. Thus, the total absolute magnitude of a given GC can be estimated by scaling the total number of giants with that in $\mathrm{M} 4$. We found $M_{\mathrm{V}}=-5.8 \pm 0.2$ for 2MASS-GC01, $M_{\mathrm{V}}=-5.6 \pm 0.2$ for 2MASS-GC02, $M_{\mathrm{V}}=-4.9 \pm 0.3$ for ESO 280-SC06, and $M_{\mathrm{V}}=$ $-6.1 \pm 0.2$ for GLIMPSE-C01. Our estimate puts GLIMPSE-C01 $\approx 2$ mag fainter than that of Kobulnicky et al. (2005), but still within their uncertainty. As a caveat, we note that the uncertainties in $M_{\mathrm{V}}$ only reflect the statistical Poisson fluctuation of the number of giants in the above GCs and M4. They do not take into account the individual spectral types or the presence of horizontal-branch stars (that are clearly seen in the 2MASS CMD of the central region of M 4 - Bonatto et al. 2007). Thus, they should be taken as lower limits on the actual uncertainties, especially in the case of ESO 280-SC06, which is $\approx 22 \mathrm{kpc}$ distant from the Sun. The GCs 2MASS-GC01, 2MASS-GC02, and GLIMPSE-C01 are only $\approx 1 \mathrm{kpc}$ more distant from the Sun than $\mathrm{M} 4$.

The resulting RDPs for the sample GCs (Sect. 4) follow the King-like profiles, which suggests that star-count losses in the central regions are not significant. Similar analysis applied to M 4 implies a loss of 2-3 giants only in the innermost region. Thus, the errors associated with such effects are encompassed by the uncertainties quoted in Table 1 (Col. 7).

In any case, the present total $M_{\mathrm{V}}$ estimates of ESO 280SC06, 2MASS-GC01, 2MASS-GC02, and GLIMPSE-C01 are consistent with the expected low-luminosity nature of these GCs (Sect. 5).

\section{Structural parameters}

Structural parameters are derived using both the stellar radial number-density and surface brightness profiles, extracted from colour-magnitude filtered photometry (Sect. 3.3).

To avoid oversampling near the centre and undersampling at large radii, the profiles are built in rings of increasing width with distance to the centre. The number and width of rings are adjusted to produce profiles with adequate spatial resolution and as small as possible $1 \sigma$ Poisson errors. The residual background level of each RDP corresponds to the average number density of colour-magnitude filtered stars measured in the comparison field. The $R$ coordinate and respective uncertainty in each ring correspond to the average position and standard deviation of the stars inside the ring. The vertical error bars correspond to the $1 \sigma$ Poisson fluctuation of the star counts in each ring. Obviously, the precision in the RDP level depends directly on the 2MASS resolution and photometric limit. Stars fainter than $J \approx 17$ and/or those with projected companions close enough to produce distorted images have been discarded by 2MASS.

The SBPs in the 2MASS $J$ band are built in a similar way to the RDPs. The average surface brightness measured in the comparison field is subtracted from what is computed in each cluster ring. As a caveat we note that the radial surface brightness is computed by summing the individual fluxes of all stars in each ring that have been resolved by 2 MASS. Thus, SBPs are essentially subject to the same technical limitations as the RDPs. In this sense, the present SBPs are somewhat different (fainter) from the classical ones built with aperture photometry.

By definition, RDPs represent star count densities, which do not take into account individual stellar magnitudes or asymmetries in the spatial distribution of stellar spectral types. Consequently, depending on the degree of large-scale mass segregation, RDP-derived parameters are expected to become larger than their counterparts obtained from SBPs.

The resulting RDPs are displayed in Fig. 4, which also shows how the residual background contamination varies in each case. Because 2MASS is magnitude-limited, the contrast with the background level and error bars among the RDPs are different and depend on the relative richness, distance from the Sun, and direction in the Galaxy of each GC. Nevertheless, most of the RDPs are well-defined with relatively high cluster/background contrasts, except for AL 3, the most centrally projected GC of the present sample.

We fit the SBPs with the empirical isothermal sphere, singlemass profile introduced by King (1962) to describe the surface brightness profiles of globular clusters. To the RDPs, we applied the King-like profile

$\sigma(R)=\sigma_{\mathrm{bg}}+\sigma_{0}\left[\frac{1}{\sqrt{1+\left(R / R_{\mathrm{c}}\right)^{2}}}-\frac{1}{\sqrt{\left.1+\left(R_{\mathrm{t}} / R_{\mathrm{c}}\right)^{2}\right)}}\right]^{2}$,

where $\sigma_{\mathrm{bg}}$ is the residual background density, $\sigma_{0}$ is the central number-density of stars, and $R_{\mathrm{c}}$ and $R_{\mathrm{t}}$ are the core and tidal 


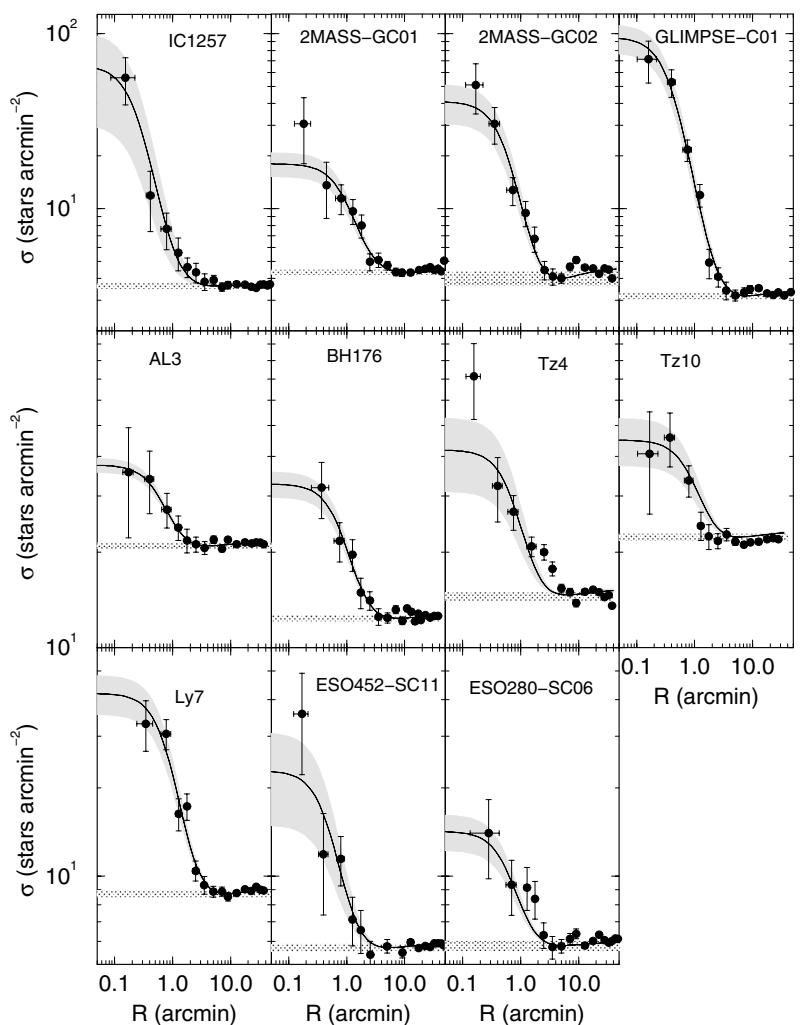

Fig. 4. Stellar RDPs (filled circles) in angular scale. Solid lines: bestfit King-like profile (Eq. (1)). Horizontal shaded region: background level $\left(\sigma_{\mathrm{bg}}\right)$. Gray regions: $1 \sigma$ fit uncertainty range.

radii. This function is similar to the King (1962) profile. In recent years, more sophisticated models have been employed to fit SBPs of Galactic and extra-Galactic GCs. The most often used are the single-mass, modified isothermal sphere of King (1966) that is the basis of the Galactic GC parameters given by Trager et al. (1995) and H03, the modified isothermal sphere of Wilson (1975), which assumes a pre-defined stellar distribution function (resulting in more extended envelopes than King 1966), and the power law with a core of Elson et al. (1987) that has been applied to massive young clusters especially in the Magellanic Clouds (e.g. Mackey \& Gilmore 2003a-c). Each function is characterised by different parameters that are somehow related to cluster structure. However, considering that the 2MASS photometric range basically covers the giant branch in most of the present GCs, and that RDP and SBP error bars are significant, we decided on the simplest model (King 1962) as fit function.

In all cases, the adopted King-like profile (Eq. (1)) represents the RDPs well along the full radial scale (Fig. 4), within uncertainties. However, the innermost point in the RDPs of Tz 4 and 2MASS-GC01 presents a $1 \sigma$ excess density over the fit, which might suggest post-core collapse. GCs with that structure occur mostly in the bulge, but some halo GCs like NGC 6397 also have it (e.g. Chernoff \& Djorgovski 1989; Trager et al. 1995). Although to a lesser extent, the same occurs for ESO 452-SC 11. Because the core radius results from the fit of Eq. (1) to the several radial points contained in RDPs, the probable post-core collapse structure of Tz 4 and 2MASS-GC01 are not reflected in the respective concentration parameters (Table 2 and Fig. 8).

Two additional parameters are computed, the half-light radius $\left(R_{\mathrm{hl}}\right)$, which can be used for comparisons with previous works and the equivalent half-star count radius $\left(R_{\mathrm{hSC}}\right)$. The

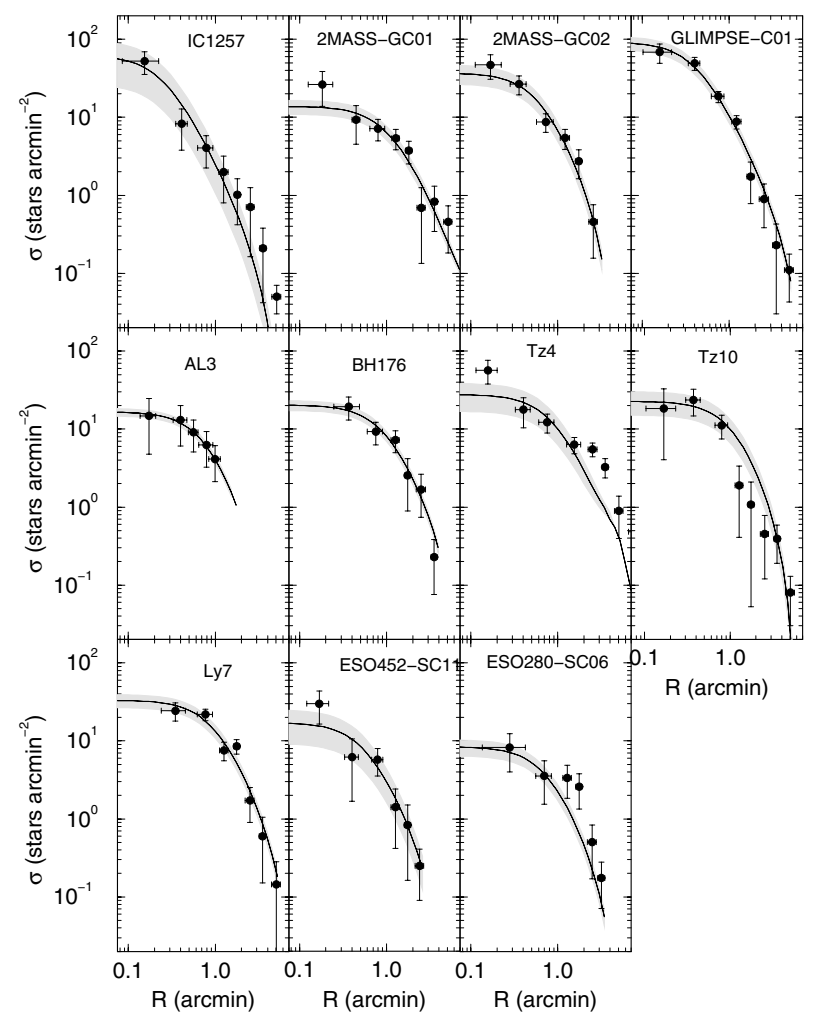

Fig. 5. Same as Fig. 4 for the background-subtracted RDPs.

half-light radius is derived through direct integration of the background-subtracted SBPs, with no fit involved in the process. Derivation of the distance from the centre which contains half of the background-subtracted cluster stars, $R_{\mathrm{hSC}}$, follows a similar procedure, where number densities are used instead of surface brightness. Figure 5 displays the present RDPs in the usual, background-subtracted presentation for GCs, which also shows the respective King-like fits and $1 \sigma$-fit uncertainties.

The RDP and SBP structural parameters that result from the above fits are given in Table 2, in angular units, where we also provide the arcmin to parsec scale for each GC, based on the distances from the Sun listed in Table 1.

Within uncertainties, both types of profiles produce similar parameters. This is confirmed in Fig. 6, where RDP and SBP parameters (in absolute units) are compared. The agreement is good for large radii, but fails for some GCs with small radii, especially the tidal one, where RDP radii are larger than SBP ones. The best overall agreement occurs for the half-type radii, and the approximate identity holds for about one order of magnitude in the radii scales (Sect. 5).

Some structural parameters, derived from SBPs, are available for GLIMPSE-C01 (Kobulnicky et al. 2005; Ivanov et al. 2005). The present values of core and half-light radii (Table 2) are comparable to those given in Kobulnicky et al. (2005) and a factor $\sim 2$ smaller than the core radius estimated by Ivanov et al. (2005). The present tidal radius, on the other hand, is $\sim 1 / 6$ of that given by Ivanov et al. (2005), which they estimated by extrapolation.

\section{Discussion}

\subsection{Photometric depth effects}

The structural parameters dealt with in this paper were derived from RDPs and SBPs built with depth-limited photometry that, 

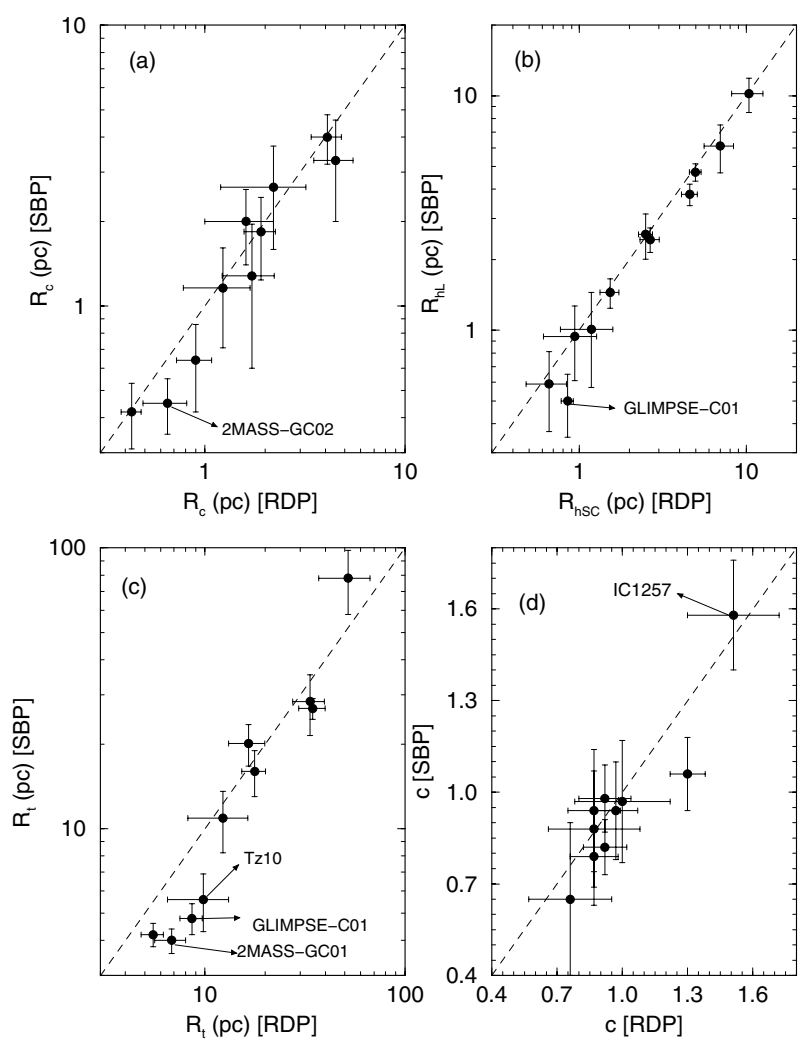

Fig. 6. Comparison of cluster structural parameters derived from RDPs and SBPs. Identity is indicated by the dashed line. GCs that lie at more than $\sim 1 \sigma$ from the identity line are labelled. The highest concentration level (panel d)) occurs for IC 1257, the most distant GC of the present sample, with $d_{\odot} \approx 25 \mathrm{kpc}$ and $R_{\mathrm{GC}} \approx 18 \mathrm{kpc}$. Absolute units are used.

for the present GCs, essentially correspond to the giant branch. Potential effects on cluster radii introduced by this observational limitation should be taken into account before comparing the parameters of present 11 faint GCs with those given in the literature for the remaining GCs that, in general, have been obtained with deeper photometry.

This issue was investigated by Bonatto \& Bica (2007b) by means of model star clusters of different ages, structures, and mass functions, with a spatial distribution of stars assumed to follow a pre-established analytical function. Model SBPs were built for the 2MASS $J$ band. Cluster radii were then measured in RDPs and SBPs extracted from data with different photometric depths, varying from the giant branch to the low-main sequence. The main results drawn from the GC models are:

(i) Structural parameters derived from SBPs are essentially insensitive to photometric depth.

(ii) RDPs built with shallow photometry yield cluster radii underestimated with respect to the values obtained with deep photometry. Tidal, half-star count, and core radii are affected with increasing intensity for a given photometric depth. Tidal, half-star count, and core radii underestimation factors are $\approx 10 \%, \approx 15 \%$, and $\approx 25 \%$, respectively, for RDPs built essentially with giant-branch stars.

(iii) Profiles with photometry deeper than the turnoff have systematically larger RDP radii than SBP ones, especially the core. In the deepest profiles, RDP to SBP radii ratios are $\approx 1.2$ both for the tidal and half-type radii, and $\approx 1.4$ for the core radius. (iv) Changes in RDP parameters with photometric depth result from a spatially variable mass function.

Most of the above results are directly related to cluster dynamical evolution. Large-scale mass segregation drives preferentially low-mass stars towards large radii (while evaporation pushes part of these stars beyond the tidal radius, into the field), and high-mass stars towards the central parts of clusters. If the stellar mass distribution can be described by a spatially variable mass function flatter at the centre than in the halo, the resulting RDP radii should be larger than SBP ones. The differences should be more noticeable in the core than the half and tidal radii, since the core would contain, on average, stars more massive than the halo, and especially near the tidal radius. Such differences, in addition, increase for profiles built with deeper photometry (Bonatto \& Bica 2007b).

It follows from the above that radial number-density and surface-brightness profiles built with shallow photometry, as is the case of the present GCs, are expected to yield similar values for the three types of cluster radii and that RDP radii are underestimated by the factors given in (ii) above with respect to the intrinsic values. However, we note that the underestimation factors for the present faint GCs are, in most cases, smaller than (or similar to) the $1 \sigma$ fit uncertainties (Table 2). The similarity between the values of RDP and SBP radii effectively occurs for most of the present faint-GC sample (Fig. 6), especially concerning the half-light and half-star count radii.

\subsection{Comparison with GCs in $\mathrm{HO}$}

In Fig. 7 we compare our faint GCs with the GCs in H03 in terms of Galactic coordinates (panels a and b), reddening $E(B-V)$ (panel c), metallicity $[\mathrm{Fe} / \mathrm{H}]$ (panel d), and distance from the Sun (panel e). The present GCs are projected not far from the bulge and close to the plane. Such difficult directions, together with relatively high reddening values in the optical (GLIMPSE-C01, 2MASS-GC01, and 2MASS-GC02 have $A_{V} \gtrsim 15$ ), explain why the present GCs have so far been scarcely studied. They have metallicities as low as $[\mathrm{Fe} / \mathrm{H}]=-1.8$ with a single case (BH 176) being metal-rich $([\mathrm{Fe} / \mathrm{H}]=0.0)$, and $\approx 73 \%$ have metallicity lower than $[\mathrm{Fe} / \mathrm{H}]=-1.0$. The same fraction of GCs is less than $\approx 10 \mathrm{kpc}$ distant from the Sun.

Further comparisons with the GCs in $\mathrm{H} 03$ are explored in Fig. 8. With respect to luminosity, most of the GCs of the present sample are $\approx 3$ mag fainter than the average luminosity of the H03 GC distribution, while at least 3 of them populate the faint-magnitude tail (panel a). The exception perhaps is GLIMPSE-C01 with $M_{\mathrm{V}}=-8.4$, but with $\approx 3$ mag of uncertainty (Kobulnicky et al. 2005). Our estimate gives $M_{\mathrm{V}}=-6.1 \pm 0.2$ for GLIMPSE-C01, which is consistent with a low-luminosity GC. About $73 \%$ of them are located within $5 \mathrm{kpc}$ of the Galactic centre (panel b), which implies that some are probably bulge GCs; the remaining GCs (IC 1257, BH 176 and ESO 280-SC06) have Galactocentric distances compatible with the halo. The present tidal radius distribution suggests a bias towards low values (panel c) with respect to H03, while the half-star count radii are more evenly distributed (panel d). The core radii appear to be distributed similarly to those in $\mathrm{H} 03$ (panel e). Consequently, the concentration parameters (panel f) are biased towards GCs of loose structure. Loose structure, together with low-luminosity $\left(M_{\mathrm{V}} \gtrsim-6\right)$, is typical of Palomar-like GCs, i.e. low-mass GCs that do not contain populous giant branches (Bonatto et al. 2007, and references therein). 

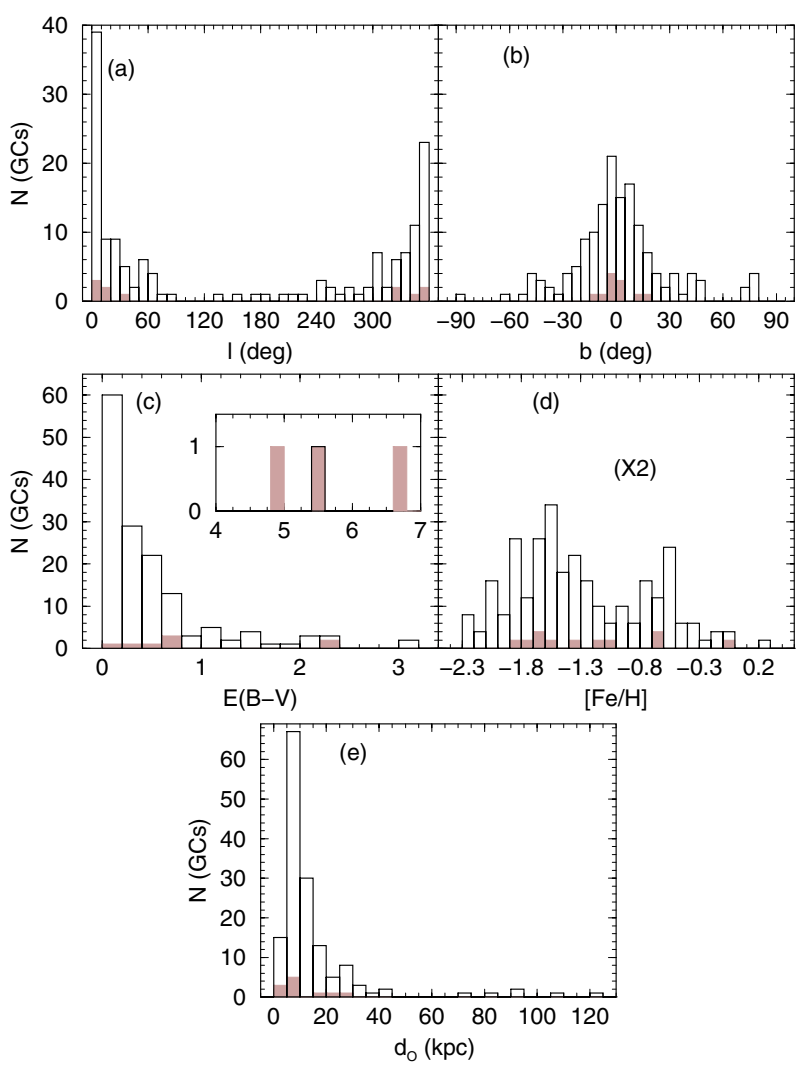

Fig. 7. Astrophysical parameters of the present faint GCs (shaded histograms) compared to the GCs in H03 (empty histograms). Inset of panel c): GCs with high reddening values. The $y$-scale in panel d) was multiplied by 2 for clarity purposes.

\subsection{Dependence on Galactocentric distance}

Finally, in Fig. 9 we examine the dependence of cluster radii on Galactocentric distance. GCs from H03 shown in this figure are separated according to luminosity, massive $\left(M_{\mathrm{V}}<-6\right)$, and Palomar-like $\left(M_{\mathrm{V}} \gtrsim-6\right) \mathrm{GCs}$. We also include for comparison the recently identified Palomar-like GC FSR 1767 (Bonatto et al. 2007). Massive and Palomar-like GCs follow the well-known relation of increasing cluster radii with Galactocentric distance (e.g. Mackey \& van den Bergh 2005; Djorgovski \& Meylan 1994). However, compared to the massive GCs, the Palomarlike ones tend to have smaller tidal and larger core radii than the massive ones for a given Galactocentric distance, especially for small $R_{\mathrm{GC}}$, which is consistent with their loose structures. The half-light radii distributions of both types of GCs are very similar.

Concerning the 11 faint GCs dealt with in this paper, the core and tidal radii distributions with Galactocentric distance are similar to those of the Palomar-like GCs. We note that this conclusion would only suffer minor changes in the case of correcting the measured radii for the photometric depth effects discussed above (Sect. 5.1). The intrinsic (i.e. corrected) core radii would be somewhat larger than the measured ones. To a lesser extent, the same applies to the relation between depth-limited and intrinsic tidal radii, thus confirming the loose structure of the present GCs.

Associated with the low luminosities (Table 1), the radii distributions with Galactocentric distance imply that the present faint GCs share similar characteristics as the sample of Palomar-like GCs present in H03.
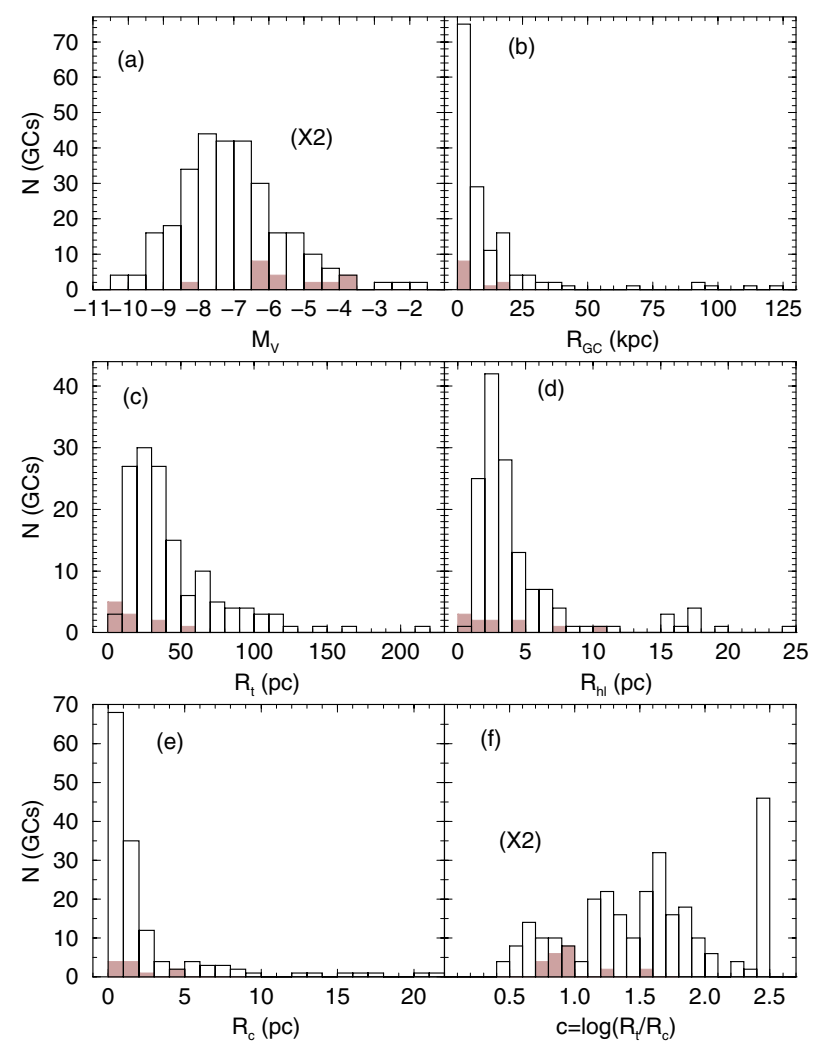

Fig. 8. Same as Fig. 7, but including the structural parameters (panels c)-f)). In panel a) we used $M_{\mathrm{V}}=-8.4$ (Kobulnicky et al. 2005) for GLIMPSE-C01; our estimate is more compatible with a lowluminosity GC, $M_{\mathrm{V}}=-6.1 \pm 0.2$. Post-core collapse GCs in H03 occupy the $c=2.5$ bin in panel f). The $y$-scale in panels a) and f) was multiplied by 2 for clarity purposes.

\section{Concluding remarks}

Compilations of astrophysical parameters of globular (e.g. H03) and open (e.g. WEBDA ${ }^{5}$ ) clusters represent a fundamental step in the study of several aspects related to the clusters themselves and the Galactic subsystems they belong to.

The majority of the GCs are rather populous and concentrated, which results in high contrast with respect to the background/foreground stellar field. The bottom line is that such GCs can be fairly easily observed in most regions of the Galaxy, from near the centre to the remote halo outskirts. Thus, through the statistics of observables such as the age, metallicity, luminosity, dynamics, and kinematics, GCs can be taken as excellent tracers of the formation history of the Galaxy. Obviously, the same applies to extra-Galactic GCs and their host galaxies.

Faint globular clusters, in particular, are important because, compared to massive clusters, they are more severely affected by the many Galaxy tidal effects and have shorter dynamicalevolution time scales. Consequently, the distribution of their structural parameters throughout the Galaxy may provide clues to the dynamical evolution of individual GCs and their system.

With the present work we derived structural parameters of 9 faint GCs listed in $\mathrm{H} 03$ and 2 others recently identified, by means of stellar radial number-density and surface-brightness profiles. These GCs either lacked such parameters or, in some cases, had first-order estimates based on CCD observations covering fields smaller than the angular diameters of clusters. The profiles in the present work were built with wide-field 2MASS

\footnotetext{
5 obswww.unige.ch/webda
} 


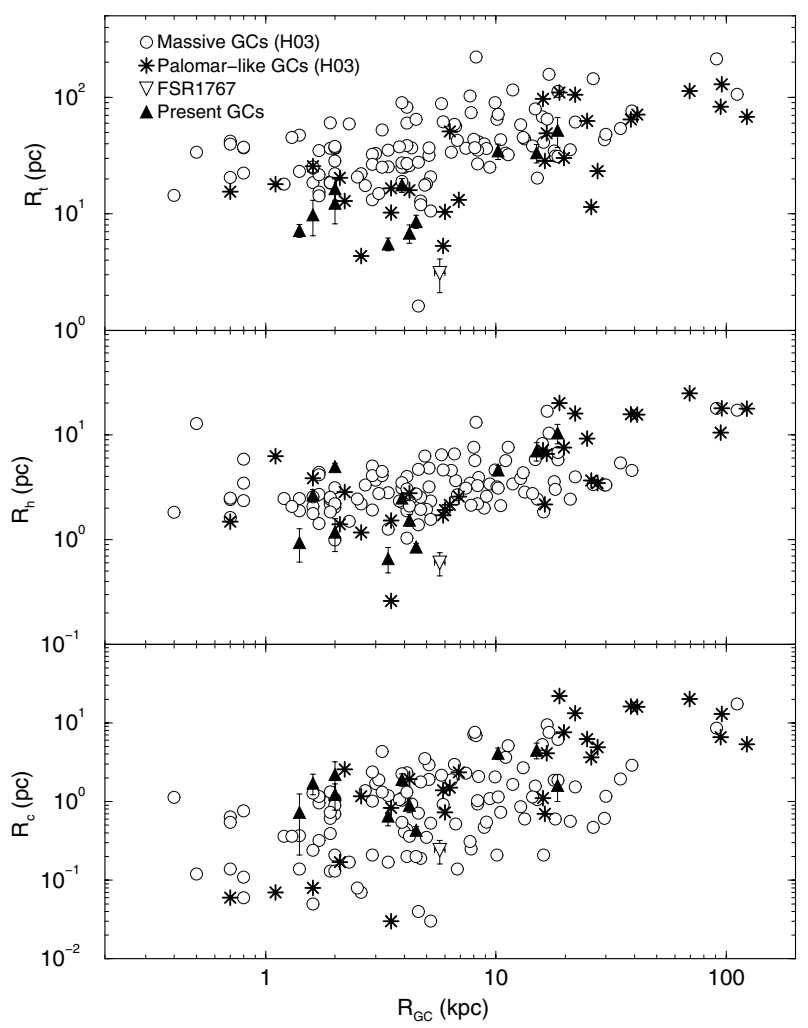

Fig. 9. Dependence of cluster radii on Galactocentric distance. Empty circles: massive $\left(M_{\mathrm{V}}<-6\right) \mathrm{GCs}$ in H03. Asterisks: Palomar-like $\left(M_{\mathrm{V}} \gtrsim\right.$ -6) GCs from H03. Empty triangle: FSR 1767. Filled triangles: present faint GCs.

photometry ( $J$ band). Because of the 2MASS depth-limit and the distances of the present GCs, the resulting photometry essentially samples giant-branch stars. In all cases, the radial profiles at least reached the respective tidal radius. We also estimated total $M_{\mathrm{V}}$ values, which were not available for ESO 280-SC06, 2MASS-GC01, and 2MASS-GC02, and provided an independent $M_{\mathrm{V}}$ estimate for GLIMPSE-C01 (which resulted $\approx 2 \mathrm{mag}$ fainter than previously given), based on the field-star decontaminated photometry of the giant stars, using M 4 as a template GC. Our estimates are in the range $-6.1 \lesssim M_{\mathrm{V}} \lesssim-4.9$, which confirms them as low-luminosity GCs.

The stellar radial profiles were fitted with King-like functions from which the core, half-star count, half-light, and tidal radii, as well as the concentration parameter, were derived. Most of the present objects turned out to be so-far neglected Palomarlike (low mass and loose structure) GCs. The distributions of core and tidal radii with respect to Galactocentric distance are also consistent with the Palomar-like nature.

Besides a better understanding of the individual structure of some faint globular clusters, this work provides an improved coverage of the GC parameter space.

Acknowledgements. We thank the anonymous referee for helpful suggestions. We acknowledge partial support from the Brazilian agency CNPq.

\section{References}

Alter, G., Ruprecht, J., \& Vanysek, V. 1970, Catalog of Star Clusters and Associations, second edition, ed. G. Alter, B. Balazs, \& J. Ruprecht (Budapest: Akademia Kiado)

Andrews, A. D., \& Lindsay, E. M. 1967, IrAJ, 8, 126

Baumgardt, H., \& Makino, J. 2003, MNRAS, 340, 227
Belokurov, V., Evans, N. W., Irwin, M. J., et al. 2007, ApJ, 658, 337 van den Bergh, S., \& Hagen, G. L. 1975, AJ, 80, 11 van den Bergh, S. 1991, ApJ, 375, 594

Bica, E., Ortolani, S., \& Barbuy, B. 1999, A\&AS, 136, 363

Bica, E., Bonatto, C., \& Blumberg, R. 2006a, A\&A, 460, 83

Bica, E., Bonatto, C., Barbuy, B., \& Ortolani, S. 2006b, A\&A, 450, 105

Bica, E., Bonatto, C., Ortolani, S., \& Barbuy, B. 2007, A\&A, 472, 483

Binney, J., \& Merrifield, M. 1998, in Galactic Astronomy (Princeton, NJ:

Princeton University Press), Princeton series in Astrophysics

Bonatto, C., \& Bica, E. 2005, A\&A, 437, 483

Bonatto, C., \& Bica, E. 2006, A\&A, 455, 931

Bonatto, C., \& Bica, E. 2007a, MNRAS, 377, 1301

Bonatto, C., \& Bica, E. 2007b, A\&A, submitted

Bonatto, C., Bica, E., \& Girardi, L. 2004, A\&A, 415, 571

Bonatto, C., Bica, E., \& Santos Jr., J. F. C. 2005, A\&A, 433, 917

Bonatto, C., Santos, J. F. C., Jr., \& Bica, E. 2006a, A\&A, 445, 567

Bonatto, C., Bica, E., Ortolani, S., \& Barbuy, B. 2006b, A\&A, 453, 121

Bonatto, C., Bica, E., Ortolani, S., \& Barbuy, B. 2007, MNRAS, 381, L45

Carraro, G. 2005, ApJ, 621, L61

Chernoff, D., \& Djorgovski, S. 1989, ApJ, 339, 904

Cohen, J. G., Hsieh, S., Stanimir, M., et al. 2007, AJ, 133, 99

Cornish, A. S. M., Phelps, R. L., Briley, M. M., \& Friel, E. D. 2006, AJ, 131, 2543

Djorgovski, S., \& Meylan, G. 1994, AJ, 108, 1292

Elson, R. A. W., Fall, S. M., \& Freeman, K. C. 1987, ApJ, 323, 54

Frinchaboy, P. M. 2006, in Globular Clusters - Guides to Galaxies, ESO/Springer Conf. Proc., ed. T. Richtler, \& S. Larsen, in press

[arXiv: astro-ph/0604133]

Froebrich, D., Meusinger, H., \& Scholz, A. 2007, MNRAS, 377, L54

Gnedin, O. Y., \& Otriker, J. P. 1997, ApJ, 474, 223

Harris, W. E. 1996, AJ, 112, 1487

Harris, W. E., Phelps, R. L., Madore, B. F., et al. 1997, AJ, 113, 688

Hurley, J., \& Tout, A. A. 1998, MNRAS, 300, 977

Hurt, R. L., Jarrett, T. H., Kirkpatrick, J. D., et al. 2000, AJ, 120, 1876

Ivanov, V. D., \& Borissova, J. 2002, A\&A, 390, 937

Ivanov, V. D., Borissova, J., \& Vanzi, L. 2000, A\&A, 362, L1

Ivanov, V. D., Kurtev, R., \& Borissova, J. 2005, A\&A, 442, 195

Janes, K., \& Adler, D. 1982, ApJ, 49, 425

Kerber, L. O., Santiago, B. X., Castro, R., \& Valls-Gabaud, D. 2002, A\&A, 390, 121

Khalisi, E., Amaro-Seoane, P., \& Spurzem, R. 2007, MNRAS, 374, 703

King, I. 1962, AJ, 67, 471

King, I. R. 1966, AJ, 71, 64

Kobulnicky, H. A., Monson, A. J., Bickalew, B. A., et al. 2005, AJ, 129, 239

Koposov, S., de Jong, J. T. A., Belokurov, V., et al. 2007, ApJ, 669, 337

Lamers, H. J. G. L. M., Gieles, M., Bastian, N., et al. 2005, A\&A, 441, 117

Lauberts, A. 1982, in ESO/Uppsala survey of the ESO(B) atlas, European Southern Observatory (ESO), Garching

Lauberts, A., Holmberg, E. B., Schuster, H. E., \& West, R. M. 1981, A\&AS, 43, 307

Lyngå, G., in Studies of the Milky Way from Centaurus to Norma. II. Open clusters, Lund Medd. Astron. Obs. Ser. II, No. 140

Lyngå, G. 1987, in Catalogue of Open Cluster Data, Lund Observatory

Mackey, A. D., \& Gilmore, G. F. 2003a, MNRAS, 338, 85

Mackey, A. D., \& Gilmore, G. F. 2003b, MNRAS, 338, 120

Mackey, A. D., \& Gilmore, G. F. 2003c, MNRAS, 340, 175

Mackey, A. D., \& van den Bergh, S. 2005, MNRAS, 360, 631

Origlia, L., \& Rich, R. M. 2004, AJ, 127, 3422

Ortolani, S., Bica, E. \& Barbuy, B. 1993, A\&A, 273, 415

Ortolani, S., Bica, E., \& Barbuy, B. 1995, A\&A, 300, 726

Ortolani, S., Bica, E., \& Barbuy, B. 1997, A\&AS, 126, 319

Ortolani, S., Bica, E., \& Barbuy, B. 2000, A\&A, 361, L57

Ortolani, S., Bica, E., \& Barbuy, B. 2006, ApJ, 646, L115

Ortolani, S., Barbuy, B., Bica, E., Zoccali, M., \& Renzini, A. 2007, A\&A, 470, 1043

Phelps, R. L., \& Schick, M. 2003, AJ, 126, 265

Sakamoto, T., \& Hasegawa, T. 2006, ApJ, 653, L29

Sarajedini, A. 2004, AJ, 128, 1228

Spitzer, L. 1958, ApJ 127, 17

Tavarez, M., \& Friel, E. 1995, AJ, 110, 223

Terzan, A. 1971, A\&A, 15, 336

Trager, S., King, I. R., \& Djorgovski, S. 1995, AJ, 109, 218

Wielen, R. 1991, in The Formation and Evolution of Star Clusters, ed. K. Janes, ASP Conf. Ser., 13, San Francisco, CA, 343

Willman, B., Blanton, M. R., West, A. A., et al. 2005, AJ, 129, 2692

Wilson, C. P. 1975, AJ, 80, 175 